\title{
A review of Electrostatic Monitoring Technology: the state of the art and future research directions
}

\author{
Zhenhua Wen*1,3, Junxing Hou ${ }^{1}$, Jason Atkin ${ }^{2,3}$ \\ (1, School of Mechatronics, Zhengzhou University of Aeronautics, Zhengzhou, 450015 China \\ 2, School of Computer Science, University of Nottingham, Nottingham, NG81BB, UK \\ 3, Institute of Aerospace Technology, University of Nottingham, Nottingham, NG81BB, UK)
}

\begin{abstract}
Electrostatic monitoring technology is a useful tool for monitoring and detecting component faults and degradation, which is necessary for system health management. It encompasses three key research areas: sensor technology; signal detection, processing and feature extraction; and verification experimentation. It has received considerable recent attention for condition monitoring due to its ability to provide warning information and non-obstructive measurements on-line. A number of papers in recent years have covered specific aspects of the technology, including sensor design optimization, sensor characteristic analysis, signal de-noising and practical applications of the technology. This paper provides a review of the recent research and of the development of electrostatic monitoring technology, with a primary emphasis on its application for the aero-engine gas path. The paper also presents a summary of some of the current applications of electrostatic monitoring technology in other industries, before concluding with a brief discussion of the current research situation and possible future challenges and research gaps in this field. The aim of this paper is to promote further research into this promising technology by increasing awareness of both the potential benefits of the technology and the current research gaps.
\end{abstract}

KEYWORDS: Electrostatic Monitoring Technology; Prognostic and Health Management; Aero-Engine;

Condition Monitoring; Electrostatic sensor; Electrostatic signal.

\begin{tabular}{|clcl}
\hline \multicolumn{2}{l}{ Abbreviations } & & \\
CBM & Condition-based Maintenance & SNR & Signal-to-Noise Ratio \\
PHM & Prognostic and Health Management & EMD & Empirical Mode Decomposition \\
UTC & United Technologies Corporation & SOA & Shaft order analysis \\
SHL & Stewart Hughes Limited & AL & Activity Level \\
IDMS & Ingested Debris Monitoring System & ER & Event Rate \\
EDMS & Engine Distress Monitoring System & HPT & High-Pressure Turbine \\
EMT & Electrostatic Monitoring Technology & AE & Acoustic Emission \\
ADC & Analog-to-digital Converter & LPT & Low-Pressure Turbine \\
DAQ & Data Acquisition & ODM & Oil Debris Monitor \\
ECG & Electrocardiography & FADEC & Full Authority Digital Engine Control \\
ICA & Independent Component Analysis & & \\
\hline
\end{tabular}

\section{Introduction}

As the heart of an aircraft, an aero-engine is a complicated system and has extremely high requirements for operational safety. Due to the limitations of the currently available materials, the design and the manufacturing process, operational

*Corresponding author.

E-mail address: levinzhwen@126.com reliability and maintainability cannot be fully guaranteed. Moreover, there are trade-offs between flight time, maintenance time and costs - e.g. more maintenance time means less time in the air. Cost and safety are key concerns for airlines. It is important to ensure economic sustainability; an aero-engine is a high-priced piece of equipment, the purchase/rental cost, the usage costs and maintenance cost are all high. The maintenance cost for the engines usually contributes up to $30-40 \%$ of the total maintenance 
cost of an aircraft.

The current progress in flight safety and flight economy for aero-engines requires improved health management capability. CBM and PHM are essential components of a health management system which is used to improve the availability and balance the economic, safety, and reliability aspects of usage and maintenance [1-7]. $\mathrm{CBM}$ tries to conduct the appropriate maintenance actions before a failure occurs, based on the currently detected condition, and is diagnostic in nature. PHM is predictive in nature and tries to predict how long from now a fault or a failure will happen. Both CBM and PHM determine the maintenance actions based on a real time or near real time assessment of health, in order to avoid unnecessary maintenance but ensure that there is sufficient maintenance to keep the aero-engine in service[8]. No matter which maintenance concept is adopted to balance the flight safety, reliability and operating profit, effective health monitoring technologies are essential in order to accurately understand the current state, track the (real time) health status, and evaluate the performance and degradation trend for the aero-engine and its component parts and subsystems. This depends on having sufficiently accurate monitoring methods, thus, condition monitoring technology is crucial to realizing the full benefits of the advanced maintenance idea and implementing a maintenance strategy $[9,10]$.

To implement the PHM concept the PHM system requires comprehensive engine information and an assessment of the engine's health, not only the current health state, but also predictions about later state, in the form of early warning information about faults and/or future maintenance requirements. Engine Health Monitoring systems acquire this information.

There is a wide acceptance of the common health monitoring methods, such as vibration monitoring, temperature detection, flow and pressure measurement at different cross sections of an aero-engine. However, although these methods can perform well for detecting some types of faults, they may not provide good fault localization.

In general, the gas path components have the highest fault rate in the aero-engine, being in such a high pressure, load, temperature and speed environment[11]. Conservative time-based preventive maintenance strategies are still a dominant maintenance policy for gas path components working near their structural limits in high-temperature environments. This policy can lead to the waste of useful residual life of the components when components are replaced before they really need to be, or may increase the risk of failure before maintenance when components degrade faster than expected, so maintenance is not soon enough. Aero-engine related incidents still occur frequently enough to indicate that the current health management systems still lack sufficient ability for adequate advance warning of faults.

The PHM concept requires engine monitoring technologies that provide early warning information about faults, and the capability to monitor the development of faults so that timely action can be taken. To achieve it, a new health monitoring technology will be required, which has the ability to provide new information, more reliable information, or earlier information than existing technologies.

Electrostatic monitoring technology for gas paths in aero-engines is one of the technologies which could provide improved prognosis abilities. As will be explained in Section 2, this technology involves monitoring the electrostatic charge to detect debris in the exhaust gas. The technology was first discovered by the Air Force Institute in the 1970s[12]. Further research on electrostatic monitoring technology was undertaken at various times by the United Technologies Corporation (UTC) [13], Stewart Hughes Limited (SHL) [14], and Smiths Industries[15] (which subsequently bought by GE ) due to licensing of the related patents and company mergers and acquisitions. SHL developed the Engine Distress Monitoring System (EDMS) and Ingested Debris Monitoring System (IDMS), as described in [16] and is discussed further in Section 5. The work on electrostatic monitoring technology by SHL and 
Smiths Industries drove the improvement and application of electrostatic monitoring technology in other industries, some of which will be discussed in Section 6.

The advantage of electrostatic monitoring technology (EMT) is that it can offer timely warning information about early faults by monitoring the change in the electrostatic level in the gas path of the aero-engines. It provides a novel method for on-line monitoring and diagnosis for components with rub faults, combustion effectiveness degradation and/or combustion chamber burnt erosion. Such faults as these cannot usually be easily detected by other current monitoring technologies [4, 12, 17-24]. Moreover, combining EMT with other existing monitoring technologies, will increase the likelihood of timely detection of faults. Thus, electrostatic monitoring technology is a useful additional tool for early detection and progression monitoring of component deterioration[25]. To underline its importance, EMT has now been as part of the Joint Strike Fighter engine prognostic health management sensor suite[4, 26].

Electrostatic monitoring technology is a wide-ranging subject and many terminologies have been used interchangeably. That these terminologies and ad hoc methods have been presented in a variety of scientific, mechanical engineering, material engineering and other publications, shows that the use of electrostatic monitoring is currently being investigated in a number of areas, such as condition monitoring for aero-engine gas path[4, 12, 14, 25-31], lubrication oil wear debris detecting[32-35], bearing wear monitoring[36-39] and flow measurement[40], rotational speed measurement[41] and particle size measurement [42], to name a few. However, it is apparent that much more research is needed into this technology, as will be discussed in the final section of this paper.

This paper reviews and discusses the current state of electrostatic monitoring technology, including sensor technology, signal detection and processing technologies, feature extraction methods, simulation experiments, existing methodologies, recent advances and remaining challenges. There is a particular focus upon the existing electrostatic monitoring technology for condition monitoring of aero-engines, however some related applications of the technology are also summarised to give the reader an overview. We end the paper with by identifying some of the remaining research gaps and less studied issues which are pertinent to the application of electrostatic sensor and discuss future avenues of research in this area.

The remainder of this paper is organized as follows: in Section 2 the principle of electrostatic monitoring technology and the formation mechanism of charged particles is explained. Section 3 provides an introduction to the sensor technology and discusses some factors which need to be considered for the optimization of the design and placement of the electrostatic sensor. The signal detection technology, processing methods and feature extraction are discussed in Section 4. Section 5 describes the related verification experiments which have already been performed for EMT. Section 6 discusses the application of electrostatic monitoring and detecting technologies to other fields. Section 7 provides concluding remarks and introduces the challenges for future research.

\section{Electrostatic Monitoring Technology}

We begin this review with a consideration of what Electrostatic Monitoring Technology actually is and how it works, along with an explanation of the source of the charged particles which are detected, and their use in identifying faults.

\subsection{The principle of electrostatic monitoring technology for aero-engines}

The fundamental principle of electrostatic monitoring technology for aero-engines is to perform real-time monitoring of the overall charge level in the gas path, on the basis that this provides insight into what is happening within the engines themselves. Generally, for a healthy aero-engine, there is a normal charge level throughout the gas path, including the exhaust pipe and intake path, and although the charge level varies with the operational condition of the aero-engine it will stay within a 
defined range. Once a fault occurs, or the performance degrades, additional debris will be present in the gas path, which leads to a change in the charge level. The normal charge level can be monitored and used to calculate a baseline reference or threshold from which subsequent deterioration in engine performance may be determined. When gas path component deterioration occurs the system detects a change in the overall electrostatic charge level [26, 28, 43-45].

The advantage of electrostatic monitoring technology is the ability to detect within the exhaust gas the direct product of the fault. The monitoring will identify the abnormal charged particles in the gas path, including both ingested objects in the inlet and the debris or particles caused by component wear or faults in the exhaust gas. This method differs significantly from common monitoring technologies such as vibration or temperature monitoring technologies, which detect the secondary effects of the fault, and the technology can be employed to detect faults earlier and provide the necessary warning information to improve the PHM capacity of an aero-engine in the overall life cycle[26, 43].

\subsection{The formation of charged particles}

Carbon dioxide, water vapor, Nitrogen Oxides, hydrocarbons, carbon monoxide, sulphur oxides, soot and debris (if a fault occurs) are the main components in the exhaust emission. The particles in the exhaust emission are electrically charged. To understand why it is worth considering how each of the types of particles is created. There are three main types of charged particles:

(1) Ions and electrons formed by the combustion chemical ionization processes in the primary combustion zone of the combustion chamber [46-48]. The main chemical reactions can be described by the following [49]:

$$
\begin{aligned}
& \mathrm{CH}+\mathrm{O}---->>\mathrm{CHO}^{+}+\mathrm{e}^{-} \\
& \mathrm{CHO}+\mathrm{H}_{2} \mathrm{O}----->\mathrm{H}_{3} \mathrm{O}+\mathrm{CO}+\mathrm{e}^{-}
\end{aligned}
$$

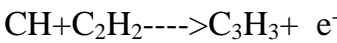

The combustion process also forms many other ionized molecules, including $\mathrm{CH}_{3}{ }^{+}, \mathrm{CH}_{3} \mathrm{O}^{+}$, $\mathrm{C}_{3} \mathrm{H}_{3}{ }^{+}, \mathrm{H}_{3} \mathrm{O}^{+}$and $\mathrm{NO}^{+}$.
(2) Soot produced by the incomplete combustion of hydrocarbons, which is usually a mass of impure carbon particles. Soot formation is a complex process and a heterogeneous interaction, where the details are still remains unclear and disputed, there has been some agreements [50-52]:

- The soot forms in the flame.

- The soot formation is related to the molar ratio of carbon to oxygen ( $\mathrm{C} / \mathrm{O}$ ratio). In premixed flames the critical $\mathrm{C} / \mathrm{O}$ ratio for soot formation is found to be about 0.5 . The $\mathrm{C} / \mathrm{O}$ ratio is closely related to the fuel type and the performance of the compressor. [12-14] analyze the charging mechanism and the factors which affect it. They clarify the effect of the fuel flow and sulfur content on the quantity of electrostatic charge and suggest that the fuel's sulfur content increases the concentration of negative ions, especially for the low flow case. Large carbon particles usually carry a positive charge, while smaller carbon particles are mainly negatively charged.

- The first step in the formation of soot particles is nucleation. The particles then continue to grow through chemical reactions at their surface, by the adsorption of the gas phase ions and molecules. Once the soot particles reach diameters in the range 0.01 to $0.05 \mathrm{um}$ they begin to coagulate to form chain agglomerates[52].

- Oxidation of the molecules and soot particles reduces soot formation.

(3) Debris caused by some fault. As the mechanical faults, the degradation or the destruction of the engine components, such as blade rubs, nozzle guide vane or turbine blade erosion and combustor burning result in the production of the charged debris $[14,16,51,53]$. The larger debris may also impact with the internal engine surfaces and interact with the charged ion or electrons. The impact process may result in an increased charge, a discharge or an inversion of the charge on the 
debris[16].

In general, the size of a soot particle in an aero gas turbine engine exhaust port is typically about $6 \mathrm{~nm}$ and the distribution of the size is a bimodal distribution with humps in the ranges of $5 \sim 7 \mathrm{~nm}$ and 20 30nm [54-56]. The sizes of carbon particles or metal abrasive and most of the abnormal particles caused by gas path component faults, such as blade rub or combustion performance degradation, are larger than 40um[14]. As explained in [57] the charging process of particles over time can be represented by formula 4 , where $q_{p}$ is the charge of the particle, $d_{p}$ is the diameter of the particle, $\mathrm{k}$ is the Boltzmann constant, $\mathrm{T}$ is the temperature, $e$ is an electronic charge, $v$ is the mean particle velocity, $N$ is the concentration (number of particles), and $t$ is time.

$$
q_{p}=\frac{d_{p} k T}{2 e^{2}} \ln \left(1+\frac{d_{p} v \pi e^{2} N t}{2 k t}\right)
$$

Based on the analysis above and Equation (4), the charging characteristic of particles is related not only to the concentration of electrons and ions but also to the size of the particles.

\section{Electrostatic Sensor Technology}

The sensors are key components and play a vital role in the monitoring system, being the source of the condition information. Sensor performance directly affects the accuracy of the early fault indicator predictions as well as the correct identification of the state of the components. This section considers the electrostatic sensors, explains how the sensors work and reviews current research in the area of electrostatic sensor design.

\subsection{The principle of an electrostatic sensor}

An electrostatic sensor is a passive sensor which is based on the electrostatic induction principle. In the electrostatic monitoring system, the sensor is used to detect the change of the electrostatic field in the sensing zone which is caused by the influence of nearby charges. The basic principle of electrostatic measuring is shown in Figure 1, which shows how the moving charge creates an electric field which is detected by the sensor[58]. The mobile charges in the interior of an electrostatic sensor probe are free to move in any direction. When a charged particle passes the electrostatic sensor, the mobile charges within the sensor move under the influence of the field from the external charge until they reach the surface of the sensor probe. The movement of charge in the sensor results in a current flow through the signal wire, and the signal can be measured by the signal conditioner. Once the charged particle moves away from the sensor, the charge inside the sensor probe returns to a neutral state.

\subsection{Sensor design}

Two key considerations for electrostatic sensor design are the material selection and the structural design of the sensor. Material selection plays an important role in ensuring that the electrostatic sensor meets the requirement to work continuously and smoothly in the high-temperature environment, not only enduring the environment, but also performing well under those conditions.

The time to reach electrostatic equilibrium can be used to evaluate the sensor performance. This time usually depends upon the conductivity of the sensor probe material [59] rather than the exerted electric field intensity or the size of the sensor probe. In general, the higher the conductivity, the less time to reach electrostatic equilibrium. In general, from a conductivity point of view, most metal materials can be used for the probe material. However, since the sensor will be used in a high-temperature environment, both the sensor probe and the isolation medium must be able to resist very high temperatures. Considering this, and the desirability for a high conductivity in the probe, Nickel alloy is usually recommended for the sensor probe and sensor cover, and Ceramic, Mica or polytetrafluoroethylene are usually appropriate for the sensor isolation medium.

In addition to the material selection, the shape of the sensor needs to be considered, to ensure that it is appropriate for different applications and different installation locations. The shape and position of the sensing surface will affect the performance. The sensitivity of the sensor and the size and shape of the space within which it is located affect the ideal sensor shape. Taking the electrostatic sensor for the gas path 
condition monitoring as an example, the ring shape is clearly appropriate for the detection of the ingested debris in the inlet[4], with its fixed position and shape, while various shapes, such as button $[14,15]$, plate $[60,61]$, cylindrical probe $[4,26,58]$, and hemisphere [62, 63], have all been proposed for monitoring the exhaust debris in the exhaust pipe. For example, [61] used a flat sensing plate-shaped sensor in the exhaust cone; whereas for other applications, a button sensor, where the probe of the electrostatic sensor has a button-shaped surface and is mounted flush in the exhaust duct or jet pipe of the engine, may be easier to install.

The limited sensing space is another factor that needs to be considered. Usually, for larger diameter gas turbine exhausts, one sensor does not have the coverage to detect particles in the entire gas path, in which case multiple sensors can be combined or linked into one compound sensor to monitor the whole section of the gas path. [64] investigated the cylindrical type electrostatic sensor arrays for the online condition monitoring.

\subsection{The electrostatic field model for sensor design optimization}

The structural parameters of the electrostatic sensor material and those of the surrounding environment have some effects on the sensitivity characteristics of the sensor at the complex electrostatic field boundary condition and on the dynamic performance, as well as the spatial filtering characteristics [58, 65, 66].

A measuring model for an electrostatic sensor is needed to describe the interaction mechanism between the moving charged particles and the electrostatic sensor. This can then be used to acquire an adequate understanding of the rules by which the structural parameters of the electrostatic sensor material affects the sensor characteristics, to provide a reference for the sensor design optimization.

Sensitivity is one of the most important characteristics which determines the dynamic performance of the sensor and the design of the corresponding signal processing circuit. The sensitivity, $S$, is usually defined by Equation 5, where
$Q^{\prime}$ is the induced charge on the sensor surface and $Q$ is the magnitude of the inducing charge, for example that charge which is carried by debris..

$$
S=\left|\frac{Q^{\prime}}{Q}\right|
$$

The main challenge for sensitivity analysis is usually, therefore, to calculate the charge induced on the surface of the sensor probe for a known charge carried by the particles.

From Gauss's law, the total electric flux through any closed surface in free space of any shape drawn through an electric field is proportional to the total electric charge enclosed by the surface, that is:

$$
\int_{V} \vec{E} \cdot d \vec{A}=\frac{Q_{\text {enclosed }}}{\varepsilon_{0}}=\int_{V} \frac{\rho}{\varepsilon_{0}} \cdot d^{3} r
$$

where $d^{3} r=d_{x} d_{y} d_{z}$ is a volume element. Based on the Divergence Theorem, Gauss's law can be expressed by Equation (7), where $\vec{\nabla}$ is the divergence operator.

$$
\vec{\nabla} \cdot \vec{E}=\frac{\rho}{\varepsilon_{0}}
$$

So the relationship between the potential $\phi$ and the charge density $\rho$ can be defined based on Gauss's law as follows:

$$
\nabla^{2} \phi=-\frac{\rho}{\varepsilon_{0}},
$$

where $\varepsilon_{0}$ is the vacuum permittivity. This relationship is a form of Poisson's equation. The induced charge $Q_{S}$ on the sensor electrode with surface area $S$ can be obtained by the following formula:

$$
Q_{s}=\int_{S} \rho \cdot d s
$$

Powrie H, Morris S etc.[36, 67] thought that not all of the charge could be detected due to the limited sensing area, so they provided an approximation calculation method for a given permittivity based on Gauss' law as the follows:

$$
Q_{S} \approx Q \cdot S / x^{2}
$$


where $Q_{S}$ is the charge induced on the sensor surface, $Q$ is the magnitude of the inducing charge, $S$ is the sensor area and $x$ is the distance between the charged particle and the sensor face.

Similarly, Liu [68] used a mathematical model based on Coulomb's law and Gauss' law to research the charge induced on the OLS sensor used in the detection of lubrication debris. This method can be used for the sensitivity analysis of a sensor for a given design.

Aiming at different industry applications, [68] also developed various electrostatic sensors and established the corresponding model to calculate the charge induced on the surface of the sensor probe by the moving charged particles. The same approach, based on the description of the electric field formed by a point charge and the electrostatic induction principle, is also used to analyze the sensing characteristics of the electrostatic sensors $[58,65,66$, 69]. The electric field formed by a point charge can be described by the following Poisson equation and Dirichlet boundary conditions[70]:

$$
\left\{\begin{array}{l}
\nabla \cdot(\varepsilon \nabla \phi(x, y, z))=-\rho(x, y, z) \\
\left.\phi(x, y, z)\right|_{(x, y, z) \in \Gamma_{F}}=0 \\
\left.\phi(x, y, z)\right|_{(x, y, z) \in \Gamma_{E}}=0 \\
\left.\phi(x, y, z)\right|_{(x, y, z) \in \Gamma_{N}}=C
\end{array}\right.
$$

where $\varphi(x, y, z)$ is the electrostatic potential; $\rho(x, y, z)$ is the charge volume density; $\varepsilon$ is the dielectric permittivity; $\Gamma_{F}, \Gamma_{E}, \Gamma_{N}$ are the boundaries of the pipe wall, sensor shield cover, and sensor electrode, respectively; and the constant $C$ denotes that the electrode is an equipotential body.

Due to the complexity of solving the Dirichlet boundary problem, the finite element method has commonly been used to establish the model for electrostatic sensors with different structures and shapes $[58,60,62-66]$, to acquire the numerical solutions. Based on the numerical analysis of the finite element model, the same authors also researched the influence on the sensitivity, the sensitivity distribution and the dynamic performance of a sensor of different factors, including the probe size, the permittivity, length and the thickness of the isolation media, and the size of the cover, etc.

[41] analyzed the factors which influence the characteristics of a cylindrical probe sensor. Figures 2 to 4 , repeated from [41], illustrate some of these factors.

Figure 2 shows how the spatial sensitivity changes with the distance to the sensor surface $d$ and the radius of the probe. From the figure it can be observed that the greater the distance of the point charge from the surface of the electrode, the less induced electric charge at the same axial position. The sensitive space can also be observed to increase with an increase in electrode radius.

Figure 3 shows how the spatial sensitivity distributions vary with the permittivity of the isolation medium. From Figure 3, we can see an increase in the permittivity of the isolation medium results in an increase in the sensitivity at the same distance $d$.

Figure 4 shows the influence of the isolation medium thickness on the sensitivity distribution. Increasing the thickness of the isolation medium causes a decrease in sensitivity.

The analysis from Figures 2 to 4 can provide a reference for sensor optimization design, but can also aid in determining the appropriate location for an electrostatic sensor. The results indicate a zone where the sensitivity is high and the variability of the sensitivity is low; this zone can be used to determine the good choices for the installation location, in order to detect more of the abnormal particles[71].

\section{Signal detection, processing and feature extraction}

The original signal from the sensor needs significant amplification, filtering and processing before it can be used. Various technologies and research approaches for analysing the data are reviewed in this section.

\subsection{Signal detection technology}

Signal detection is the process of measuring and storing information from the sensor output for the purpose of providing warning and diagnostic information. 
The measurement system, to which the probe sends the signal, is responsible for detecting, measuring and storing the measurements in the condition monitoring system.

An electrostatic monitoring system usually consists of a preamplifier, filter, ADC (Analog-to-digital Converter), DAQ (Data Acquisition) card and data processor. The preamplifier is used to amplify the electrostatic inducing signal to a measurable voltage, since the inducing signal will usually be extremely weak. The resulting voltage signal is then fed into the ADC and the output is passed to the acquisition system and stored in the computer for further analysis and processing.

Since both the velocity of the charged particles and the flow characteristics have effects on the frequency characteristic of the electrostatic signal, an analysis of frequency domain features forms the basis for the design of a charge amplifier and the related signal processing circuit.

Both the theoretical analysis and the experimental results in the literature $[58,72]$ show that an increase in the moving velocity of the charged particles results in an increase in the high-frequency content in the signal, and the larger the continuous particle flow, the broader the frequency spectrum range.

In exhaust gas, fine particles, such as soot, smoke, and smaller debris, usually move faster than larger debris derived from faults. Thus, higher frequency signals indicate that finer or faster moving particles are present in the gas path. In contrast, low-frequency content is informative for judging the quantity of abnormal particles or large-sized debris present in the sensing zone. Therefore, maintaining the low-frequency response is crucial to the design of the measurement system, especially the preamplifier.

Addabbo et al [73, 74] presented a charge amplifier whose frequency response can be specifically tailored to the current application and proposed a detection post-processing algorithm to estimate the shape of the input charge pulse. This amplifier can be used to detect ultra-slow moving charged particles. Although there are significant differences between the velocities of particles in the experimental situation and the real aero-engine gas path, the analysis method and amplifier design still provide useful references for the measuring system.

\subsection{Signal processing}

Noise reduction is the process of removing noise from the original signal. This paper will not cover the details of how signal processing techniques work, but the authors would like to note that de-noising is essential for extracting the effective features after detecting the induced electrostatic signal. The purpose of de-noising is to improve the signal-to-noise ratio (SNR), ensuring that the extracted features are appropriate for diagnostic and prognostic purposes and improving the accuracy of the early warning information and fault classification.

Various signal processing techniques have been developed to remove the noise from the vibration or other acoustic emissions signals, which have various differing noise characteristics. These methods include cut-off frequency methods based on the Fourier transform, wavelet de-noising methods, etc. Because of the specific characteristics of electrostatic signals, such as being non-stationary, having a continuous frequency spectrum distribution, or having a varied distribution of frequency spectrum under different working conditions [58], the simple cut-off frequency method is not suitable for processing this kind of signal. However, the studies on time-frequency and time-scale analysis methods show promise for processing it. Wavelet transform techniques [75-77] have been successfully applied to process non-stationary signals in the past, such as the vibration signal of gear boxes[78], bearings [79] and other rotary mechanical systems [80]. Moreover, from the shape of the output signal (see Figure 1), the electrostatic monitoring signal can be observed to be similar to the electrocardiography (ECG) signal [81]. So, the de-noising methods based on time-frequency and time-scale analysis which have been used for ECGs are also promising for the electrostatic monitoring signal processing.

Nevertheless, the type of the source noise which 
is mixed into the electrostatic monitoring signal, in terms of both strength and distribution, needs to be considered when selecting de-noising methods to be employed. For instance, the noise in the electrostatic signal acquired from the experiment platform is a mixture of random noise and impulse noise, which can be difficult to remove from the original signal. Addressing this problem, [82]proposed a hybrid de-noising method which combines the wavelet transform and median filter method to remove the noise in the electrostatic monitoring signal. As reported in [82], the median filter method was used as a smoothing method to preprocess the signal, using the following principle:

(1) First define a step size, $s$

(2) Next define a sliding window of specified length in multiples of the step size (length=x.s).

(3) The first $x-1$ windows will not have enough data, so the algorithm has to determine what to use for these missing values. Common approaches include repeating the first element or using a zero value.

(4) Calculate the median of the data in the window.

(5) Move the window by the step size and repeat step (3) until the end of the sample is reached.

(6) The median of each window is used as the output, being the original signal preprocessed by the median filter method.

In[82], the preprocessed signal was then further de-noised by the wavelet transform method based on the Birge-Massart strategy. In this way, the noise which was mixed in with the electrostatic signal was effectively removed.

[83] applied independent component analysis (ICA) to remove the noise from the electrostatic signal. ICA is a computational method for decomposing a multivariate signal into independent non-Gaussian signals. It has been commonly used in the field of blind source separation. It is based on two assumptions: the source signals are independent of each other and the values in each source signal have non-Gaussian distributions. The ICA-based de-noising method requires that the number of source signals should be more than the number of independent output signals. For practical applications of the electrostatic monitoring signal, however, the source signal is usually one-dimensional, so the authors of [83] employed the Empirical Mode Decomposition (EMD) technique to decompose the original signal into multiple empirical modes, then, on the basis of frequency and wave shape, selected some mode functions from the decomposed components to construct the reference noise signal, before finally using the FASTICA algorithm to separate the noise from the electrostatic signal.

Measuring the performance in terms of the improvement in the SNR of the signals, the methods mentioned above were shown to perform well and have a good ability to remove noise[82, 83].

\subsection{Feature extraction}

In the condition monitoring system, pre-warning information should be triggered when features exceed threshold values. Tracking the key features of electrostatic monitoring signals can also provide a base reference for the condition evaluation of components, or the entire system, in the gas path of the aero-engine. For feature extraction for the electrostatic signals, the features are usually divided into two classes: the time domain features and the frequency domain features:

\section{(1) Time domain analysis:}

The most common time domain features include the amplitude, range, RMS, MAX, and MIN values. Two further important time domain features are also commonly included: Activity Level $(A L)$ and Event Rate $(E R)$, which are both explained below.

Assume that $N$ samples are available for time $t$. $A L(t)$, the activity level at time $t$, is a measure of the amplitude of the samples and can be described by Equation (12) where $Q_{n}$ is the charge of the $n^{\text {th }}$ sample.

$$
\begin{aligned}
& A L_{T}(t)=\sqrt{\frac{1}{N} \sum_{n=1}^{N} Q_{n}^{2}} \\
& E R_{T}(t)=\frac{M}{N} \cdot 100 \%
\end{aligned}
$$

$E R(t)$, the event rate for time $\mathrm{t}$, is a measure of the percentage of samples which exceed some multiple, 
$K$, of the activity level. i.e. $E R(t)$ assesses the number of samples for which the charge is unusually high, indicating the presence of abnormal particles. $\operatorname{ER}(t)$ is described by Equation (13) [21] where $M$ is the number of the $N$ total samples available for time $t$ for which $\left|Q_{n}\right|>K \cdot A L$, where the constant $K$ is usually obtained from an evaluation of experimental and statistical data and will differ depending upon the threshold required for the current working conditions.

Generally speaking, the Activity Level can be used to give an indication of the amount of fine carbon particles or debris in the exhaust gas. Because these small charged particles are the main content of the exhaust gas, they contribute the majority of the overall charge level, and the charge level caused by the fine carbon particles is usually used to calculate the baseline charge.

The Event Rate relates to the proportion of large particles which are present in the gas path per unit time. The events can also be separated according to their polarity into positive and negative events. Hence, combining this information, AL and ER can be used to identify large particles and small particles, and the polarity can be used to discriminate between carbon particles and metal debris[14, 84]

\section{(2) Frequency domain analysis:}

The frequency content of the signal is related to the velocity of the charged particles, the size and the flow characteristics, etc., so the frequency features can also give an indication of the current working condition of the components in the gas path. This is especially useful for identifying whether abnormal particles are caused by static or rotary part faults.

The frequency spectrum distribution of the electrostatic signal particles is continuous, as shown in Figure 5, although it can also be observed that there can be differences in the shape of the signals based upon factors such as the velocities of the charged particles.

It is also shown in[58] that differences in frequency spectrum are detectable for charged particles with different flow characteristics, such as their velocities, the continuous distribution or the discrete distribution of charge particles. This will be discussed further in Section 5.

The maxima in the frequency spectrum will depend upon the characteristics of the sensor and measuring system. Therefore, in the process of extracting the frequency domain features, peak detecting technology is applied to find the maxima in the frequency spectrum and record the corresponding frequency value $f_{\max }$, which is often called the characteristic frequency. The frequency of the peak will increase with the velocity of the charged particles, however. The existence of a similar maximum frequency $f_{\max }$ was also found in [85], where it was named VI/VII by the authors.

From the frequency spectrum analysis of the electrostatic signal in [58] there are obvious differences in the distribution structure of the frequency spectrum for the signals caused by different sizes or velocities of particles. Hence, the signal can be decomposed using the wavelet analysis method into different frequency bands and then the energy distribution of the signal in different frequency bands can be calculated[72].In the process of wavelet decomposition, re-sampling is needed. The re-sampling frequency $f_{s}$ can be set such that $f_{s}=A \cdot f_{\max }$, where $\mathrm{A}$ is a positive integer constant, in order to remove the influence of the velocity of the charged particles, and the characteristic frequency $f_{\max }$. The energy distribution characteristics can then be employed to identify whether the additional charged particles are caused by static or rotary component faults within the engine[72].

In addition, Shaft Order Analysis (SOA) was used in $[16,25,30]$ to judge whether gas path debris generation correlates with any of the spool speeds, by analyzing the frequency spectrum of the electrostatic signal, e.g. to identify whether the fault is with rotating parts, such as a rub fault.

\section{Experimental Verification Research}

The current verification experiments for electrostatic monitoring technology can be divided into three classes.

The first class of experiments consists of 
simulation experiments for the initial basic research into electrostatic monitoring technology. For example, the verification experiment for the charging mechanism of particles, the qualitative flexibility analysis of the electrostatic sensor and electrostatic monitoring technology, and for verification of the effectiveness of the sensor and the features of the signals. For example, [44, 45] built an experimental platform to simulate the combustion environment, and fed in abnormal metal particles to test the electrostatic sensor. The results showed the obvious change of the output signal from the electrostatic sensor, testing the effectiveness of the electrostatic sensor and the electrostatic monitoring technology. Fig 6 shows the amplitude of the electrostatic signals over time for this experiment. The experiments involved adjusting the gas-oil ratio and later discontinuously seeding with metal particles of size 75 um. The resulting carbon particles from the ratio change were detected as a change in amplitude around time $20 \mathrm{~s}$ to $40 \mathrm{~s}$, and the metal particles were detected as the amplitude change around 90s to $110 \mathrm{~s}$.

In [85], the authors stated that there were two different particle sources. The first set of particles were continuously produced by the combustion, and are usually continuously present in the jet pipe. The second set of particles consists of abnormal particles which discontinuously appear as a result of damage. These latter charged particles move as separate clusters, rather than a continuous stream. For this reason, the authors of [85] developed experimental facilities (a subsonic turbulent vapor-air jet, a corona and a Taylor cone) for the simultaneous simulation of both the continuous and discontinuous regimes of charged particle motion.

The experiment showed that two power frequency maxima were detected, related to a continuous distribution of small charged particles and a discrete distribution of large charged particles, respectively.

The second class of experiments explored these relationships between the electrostatic signal and the health status of the engines. The experiments were usually conducted in test rigs on the ground, which were used to explore the change rules for the electrostatic monitoring signals with the performance or fault occurrence in the gas path.

In fact, electrostatic monitoring technology originated from an experiment by the Air Force Institute of Technology (AFIT). They discovered that some jet engine gas-path failures are preceded by an increase in electrical activity in the jet exhaust gases [23]. [24] also found that the electrical activity in the exhaust gas increases before engine gas path failure. At that time, there was only statistical evidence that pockets of charge preceded some engine failures. Dunn and Mitchell found, in the experimental process of rubbing and burning, that large amounts of metal particles (size: 5-100 $\mu \mathrm{m}$ ) fall off from the surface of the gas path components when rub and burn erosion occurs, which leads to an increase in charged particles in the gas path. In further research, the quantity of charged particles and the amplitude were observed to have a similar trend to the degradation trend [17, 24]. Couch et al [86] developed an apparatus for the application of electrostatic monitoring technology to the exhaust gas of the jet engine. In this setup, an electrostatic probe is used to detect the ion current and a trigger circuit is applied to provide the warning information. Couch[87] also performed research which verified that the performance degradation of a turbine engine can be detected from the electrostatic pulse signals. Approximately two out of three turbine engine problems could be predicted four or more hours ahead of failure using the electrostatic monitoring technology, and the false alarm rate was only about $5 \%$.

[14] tracked the relationship between the working condition and the electrostatic signal using accelerated life experiments for a turbine gas engine. The experimental results showed that the activity level and the event rate both changed under different conditions and verified the feasibility of providing early fault warning information for issues such as a rub fault in the compressor or a fault in the combustion chamber. The technology was proposed to be employed to enable overall prognosis and health 
management for aero-engines.

$[25,30]$ processed the EDMS Data and calculated the signal components such as the activity level, event parameters and the shaft order energy, which can be used as indicators for High Pressure Turbine (HPT) erosion, Low Pressure Turbine (LPT) rub, blocked Nozzles and augmenter faults.

[88] found that water washing, oil leakage and combustor linear cracking of an aero-engine can all be detected using the electrostatic monitoring method. [84]tracked the relationship between the output power and the electrostatic signal using an accelerated life test experiment on a turbo-shaft engine.

In one test cycle, the engine experiences 14 working conditions in a specified order, each of which lasts for a specified time, while the DAQ system simultaneously records the electrostatic monitoring signal[84]. Figure 7 shows the output power of the engine for an example test cycle, while Figure 8 shows how the amplitude of the electrostatic signal changes with the output power of the turbo-shaft engine. From Figure 8, we can see that the electrostatic monitoring technology can effectively reflect the change of the output power of the engine.

The third class of experiments is on-board experimentation for verification of the technology. In these, the practical application is investigated and high altitude experimentation is performed to collect the data to verify the algorithms, sensor installation, various features of the signals, validity of the electrostatic monitoring system, and so on. As mentioned in Section 1, SHL developed the IDMS and EDMS systems based on electrostatic monitoring of the charged debris in the gas path. IDMS is used to detect a foreign object entering the engine via the intake, while EDMS is used to real-time monitor gas path component degradation. The hardware configuration, technology maturity and the applications for IDMS/EDMS were presented and discussed in $[4,26]$. The integration of IDMS and EDMS has allowed the impact of foreign objects ingested into the engine to be analyzed and identified
[16].

\section{Other applications of electrostatic monitoring technology}

Due to its non-destructive nature and the pre-warning capability, electrostatic monitoring has been popular for applications in monitoring and measurement in other fields as well. There are many links between the fields as well as distinct differences in many cases, and it is possible that breakthroughs in one field could lead to benefits in others. For this reason, the following section presents some of the other related applications of electrostatic monitoring technology which it is useful to be aware of.

\subsection{Wear debris detection in Lubrication Oil}

Wear is the most common type of mechanical problem, and the detection and analysis of particles within lubrication oil is a general approach for detecting this, measuring the shape or content of particles in the lubrication oil.

There are four commonly used oil analysis methods: Ferro graphic analysis based on the size and shape of the debris; spectral analysis for measuring the contamination of metal elements in the lubrication oil; physicochemical analysis for detecting the physical properties and chemical properties such as the acidity test; and contamination analysis, counting the big particles per unit volume of oil. All four of the methods have been commonly used in the aeronautical industry for wear fault diagnosis and prognosis, but these oil analysis methods are usually performed offline, with the analysis being done somewhere else, usually in an oil analysis laboratory. The results then turn up later so do not really reflect the real-time status, leaving the existing methods with a shortfall in temporality. Moreover, these methods usually have a more limited range of detectable object sizes.

An oil debris monitor (ODM) for these problems was jointly developed by BFGoodrich Aerospace and GasTOPS, which overcomes the limitations of conventional chip detection systems. The ODM system uses an advanced, full-flow, inductive sensing technology which is capable of continuously monitoring metal particles from wear, both 
ferromagnetic and non-ferromagnetic. The ODM sensor is designed to be installed into the existing chip detector part. However, due to the ODM detector chip limitations, there is only a low capture efficiency; ODM is not sensitive to smaller particles or debris sizes: less than $100 \mathrm{um}$ for ferrous particles and $250 \mathrm{um}$ for non-ferrous particles [89-92]. The lack of prediction of the trend in the amount of debris over time, and the insensitivity to smaller non-ferrous debris are some of the other possible drawbacks.

Due to the poor timeliness of off-line analysis and the limitations in the detectable range of the current monitoring techniques, wear faults are often not detected in a timely manner, thus wear faults still account for a large proportion of mechanical faults in aero-engine operation. Therefore, researchers have investigated the application of electrostatic monitoring to lubrication oil debris detection, measuring the charged wear debris, detecting the direct product of the fault [34, 68, 93-96]. Potential sources of charged debris include surface charging, triboemission when the component surface is damaged, and the breaking of multiple atomic bonds when the debris is produced[67, 94].

A ring-shaped electrostatic sensor is used in the lubrication oil monitoring system and is mounted on the oil pipe, monitoring the debris in that whole section of the oil pipe. It is also called an OLS (oil-line sensor) and, being ring-shaped, it has similar characteristics to the electrostatic sensor in the intake of an aero-engine. Powrie et al[96] used the OLS to perform real-time monitoring of the oil-borne debris and found that the electrostatic charge signals can be taken as an early indication of component degradation and increased generation of wear debris.

$[34,67,94,96]$ showed the sensing capabilities of the electrostatic monitoring technology for both oil-line and wear-site debris in one monitoring suite by utilizing an accelerated bearing failure test under both overloaded and seeded fault conditions.

\subsection{Wear site detection for rolling bearing}

The main monitoring method for wear debris detection in lubrication oil is to detect the product of the wear fault debris, as mentioned above. However, an electrostatic sensor could also be used to monitor the wear site directly. In the process of contact wear, tribo-charging, tribo-chemistry, contact charging, tribo-emission and contact potential difference are all sources of electrostatic charge, so the electrostatic sensor can be applied directly to monitor the charge on the wear site[34, 36-39, 67].

Harvey [34] employed an electrostatic monitoring system to detect the onset of wear in lubricated tribo-contacts, in order to detect the early stages of the components' surface breakdown and wear. They mounted the electrostatic sensor close to the bearings in their experiment. [37] used an Acoustic Emission (AE) sensor and electrostatic sensor (ES) to monitor the various phases of delamination wear for a bearing steel contact under dry sliding conditions. Both the $\mathrm{AE}$ and $\mathrm{ES}$ monitoring results were found to correlate with friction levels and wear rates.

\subsection{Mass flow rate and rotary speed measurement}

In addition to its application for condition monitoring, another similar application of electrostatic monitoring is mass flow rate measurement in the gas-solid two-phase flow. This is required in many industries, such as within power plants or steel production [40, 97, 98].

Here we use the power plant as an example to illustrate the application. In general, the pulverized coal concentration is one of the most important parameters in the monitoring system for the power plant. This can be acquired by measuring the velocity of the pulverized coal. Due to the electrification by friction between the pulverized coal and the pipe, a pair of electrostatic sensors can be used to detect the resulting electrostatic charge and calculate the time delay between the two signals based on a cross correlation algorithm. From this, an estimation can be made for the velocity and concentration [99-104].

Similarly, the rotational speed can be one of the more important parameters for a condition monitoring system to determine when controlling a rotary machine, and an electrostatic sensor can aid here as well, sensing the movement of the surface using electrostatic induction. Similarly to the velocity 
measure for mass flow, the sensing principle makes use of the phenomenon that a rotating device generates an electrostatic charge on its surface due to air friction.

The main difference between the mass flow and rotary speed techniques is that the two sensors are mounted with a fixed linear distance for mass flow measurement, but fixed angles for rotational speed measurement.

[41] employed electrostatic sensors to measure the movement of a rotating surface and compute the speed based on the cross-correlation algorithm. In their facility, an array of electrodes which was placed adjacent to the rotating surface was employed to sense the movement of the surface through electrostatic induction. The rotational speed could then be determined through the cross-correlation of the signals from the electrodes. The experimental results showed that the maximum error was no greater than $\pm 2 \%$ under all test conditions, over the speed range of 0-3000 r/min.

Using an electrostatic sensor for measurement provides a noncontact measurement, with low cost, which is suitable for condition monitoring application even in hostile environments.

\section{Conclusions and future work}

In this work we have attempted to summarize the recent research and development in electrostatic monitoring technology, emphasizing aero-engine health management, but also discussing some of the related applications within other industries since ongoing research may have benefits beyond those industries. This review has covered the underlying principles of electrostatic monitoring technology, the detection technologies, the measurement models and signal processing, and the main feature extraction algorithms.

Although alternative advanced online condition monitoring technologies have been available for a while, such as vibration or temperature monitoring, and have been applied with good results in many situations, there are situations where they may not be appropriate, such as when there is limited structural space, a hazardous environment and/or the warning information is urgently needed in real time to ensure safety. In these cases, the classical sensors may not work as efficiently, and an electrostatic monitoring technique can be a much better choice.

A novel condition monitoring technology with prognostic ability will focus on providing appropriate warning information before failure occurs, for the purpose of system health management. Despite the past and ongoing work in electrostatic monitoring techniques, we believe that there are still open questions and that the following research directions need to be addressed if electrostatic monitoring techniques are to be fully utilized for PHM:

1. Considering sensor technology, research is needed to minimize the size of the sensor and to optimize the sensor design in order to better adapt to the hostile working environment, as well as to improve the resolution of the sensor and the sensing scope. The obvious conflict between a limited detection scope for the electrostatic sensor and a larger diameter engine exhaust or oil pipe needs to be solved, although multiple electrostatic sensors have been tentatively applied in some cases to address the problem.

2. EMT is a relatively new technology compared with many of the technologies in use. It is clear from the state of the art at the moment that further work is needed on the integration of the technology within current health monitoring systems, including work on hardware integration and information fusion. In this context, hardware integration mainly involves integrating the electrostatic monitoring technology into the Full Authority Digital Engine Control (FADEC) system. Information fusion needs to consider the integration of different information, such as from multiple electrostatic sensors as well as between the electrostatic monitoring signals and condition monitoring information from other sensors, such as those monitoring the rotation speed of each spool, measuring vibrations, fuel flow etc. The introduction of electrostatic monitoring technology broadens the range of the component condition information and could increase the confidence in 
fault prognosis and diagnosis given a novel information fusion mechanism to make full use of the condition monitoring information.

3. The lack of baseline and threshold values for aero-engines under various working conditions, including different working environments, engine conditions and fuel details, will need resolving and more work is needed in order to produce this information. Seeded fault experiments, simulated fault experiments, accelerated life experiments and performance tracking experiments will all need to be performed to verify the appropriate baseline and threshold values for each feature of the electrostatic monitoring signal. A dynamic baseline and threshold could be advisable depending upon the situation and maintenance history. Work should be put into designing the experimental program, the data processing and the data mining methods to determine these.

4. Prediction models are needed which can predict the remaining life based on the electrostatic monitoring information. There are open questions, such as how far in advance the warning information can or should be provided, how to adjust or preset the warning time based on the maintenance strategy, and what is the actual shortest response time? It should be noted that electrostatic monitoring system has not been fully optimised and future developments to increase the sensitivity to system performance deterioration are expected in the future. These may further affect the models.

5. Evaluation work will be needed to assess the effect of introducing the electrostatic monitoring technology, including any change in the reliability of the monitoring system, and the implementation costs compared with the return on investment from any increase in prediction accuracy from the new sensors.

In summary, we believe that electrostatic monitoring technology is a promising technology, which is worthy of more research to advance both the hardware and prediction techniques. Although there was some research around the turn of the century, there was been only limited research, in very few locations, in the last few years. We believe that this may be unnecessarily restricting the growth and breadth of application areas for this technology. This paper has aimed to promote this research by providing an accessible overview of the technology, how it works, the currently employed techniques, and the current state of the art of the research in this area.

\section{Acknowledgements:}

The work is supported by the Chinese Scholar Council (201608410051) and partly supported by the foundation of Henan educational department for the Young Core Teachers in University (2014GGJS-104) and the key project of the education department of Henan Province, China (14A590001,17A470004). The authors wish to thank these organizations for their financial support!

\section{References:}

[1] Hess A. The joint strike fighter (JSF) prognostics and health management. NDIA Conference 2001.

[2] Hess A, Calvello G, Dabney T. PHM a key enabler for the JSF autonomic logistics support concept.Aerospace Conference, 2004 Proceedings 2004 IEEE: IEEE; 2004.p.3543-3550.

[3] Hess A, Fila L. The joint strike fighter (JSF) PHM concept: potential impact on aging aircraft problems. Aerospace Conference Proceedings, 2002 IEEE: IEEE; 2002.p.6-3021-3026-3026 vol. 3026.

[4] Novis A, Powrie H. PHM sensor Implementation in the real world-a status report. Proceedings of IEEE Aerospace Conference. USA: IEEE; 2006.p.1-9.

[5] Wen Z, Liu Y. Applications of Prognostics and Health Management in aviation industry. 2011 Prognostics and System Health Management Conference: IEEE; 2011.p.1-5.

[6] Cutter DM, Thompson OR, Logistics Management Inst Mclean VA. Condition-Based Maintenance Plus Select Program Survey: Defense Technical Information Center; 2005.

[7] Navarra K, Lawton R, Hearrell N. An Enterprise Strategy for Implementing Conditioned-Based Maintenance Plus (CBM+) Research in the USAF. IEEEAC paper2007.p.1-7.

[8] Das S, Hall R, Patel A, McNamara S, Todd J. An 
open architecture for enabling CBM/PHM capabilities in ground vehicles. Prognostics and Health Management (PHM), 2012 IEEE Conference on: IEEE; 2012.p.1-8.

[9] Carter MC, Kennedy JS. Cost-wise readiness enabled through data driven fleet management (DDFM): Measuring PHM system benefits through post implementation assessment. Prognostics and Health Management (ICPHM), 2016 IEEE International Conference on: IEEE; 2016.p.1-8.

[10] Smith CT. USAF Condition Based Maintenance Plus (CBM+) Initiative. Air Force Logistics Management Agency 2003.

[11] Clifton DA, Tarassenko L. Condition monitoring of gas-turbine engines. Transfer Report, Department of Engineering Science, University of Oxford2006.

[12] Couch RP, Rossbach DR. Sensing Jet Engine Performance and Incipient Failure with Electrostatic Probes. DTIC Document; 1972.

[13] Rosenbush DM, Couch RP. Electrostatic engine diagnostics with acceleration related threshold. U.S.Patent; 1986.

[14] Powrie HEG, K.McNICHOLAS. Gas path condition monitoring during accelerated mission testing of a demonstrator engine. The 33rd AIAA/ ASME / SAE/ASEE Joint Propulsion Conference and Exhibit. Seattle,WA,USA: AIAA; 1997.p.2904.

[15] Powrie H, Worsfold J. Gas Path Debris Monitoring For Heavy-Duty Gas Turbines-A Pilot Study. IDGTE Gas Turbine Symposium2001.p.168-179.

[16] Angello L. Combustion turbine electrostatic debris monitoring system (EDMS) assessment. Electric Power Research Insitute EPRI, Tech Rep 2004.

[17] Dunn RW. The Electrostatic Sensing of Simulated MA-1A Gas Path Distresses: DTIC Document; 1976.

[18] Gifford III WE. A Method for Analysis of Electrostatic Probe Signals Relating to Jet-Engine Microdistresses. DTIC Document;
1976.

[19] Murnane SN, Barnes RN, Woodhead SR, Amadi-Echendu JE. Electrostatic modelling and measurement of airborne particle concentration. IEEE Transactions on Instrumentation and Measurement 1996;45(2):488-492.

[20] Dunn R, Wright A, Hudson M. Charged particle detection in rocket plumes for health monitoring. AIAA/ASME/SAE/ASEE Joint Propulsion Conference \& Exhibit, 34 th, Cleveland, OH1998.

[21] Wen Z, Zuo H, Pecht MG. Electrostatic monitoring of gas path debris for aero-engines. IEEE Transactions on Reliability 2011;60(1):33-40.

[22] Fu Y, Zuo H, Wang R, Liu P, Cai J, Wen Z. A Monitoring Experiment for Gas Path Electrostatic Probe-type Sensor on Turbojet Engine. Information Technology Journal 2013;12:331-337.

[23] Couch R, Rossbach D, Burgess R. Sensing incipient engine failure with electrostatic probes. Instrumentation for airbreathing propulsion 1974:515-529.

[24] Mitchell JE. Exploding Wire Simulation of Jet-Engine Gas-Path Microdistresses: Air Force Inst of Tech Wright-Patterson AFB OHIO School of Engineering; 1975.

[25] Fisher CE. Gas path debris monitoring-A 21st century PHM tool IEEE Aerospace Conference Proceedings. Montana, USA: IEEE 2000.p.441-448.

[26] Powrie H, Novis A. Gas path debris monitoring for F-35 joint strike fighter propulsion system PHM. Proceedings of IEEE Aerospace Conference. Montana, USA: IEEE; 2006.p.1-8

[27] Powrie HEG, Fisher CE. Engine Health Monitoring Towards Total Prognostics. IEEE Aerospace Applications Conference Proceedings. CA,USA: IEEE; 1999.p.11-20.

[28] Powrie HEG, Fisher CE. Monitoring of Foreign Objects Ingested into the Intake of a Gas Turbine Aero-Engine. International Conference on Condition Monitoring 
Proceedings. UK: University of Wales Swansea1999.p.175-190.

[29] Powrie HEG, Worsfold J. Gas Path Debris Monitoring For Heavy-Duty Gas Turbines-A Pilot Study. IDGTE Gas Turbine Symposium2001.p.168-179.

[30] Fisher C. Data and information fusion for gas path debris monitoring. IEEE Aerospace Conference Proceedings. Montana, USA: IEEE; 2001.p.3017-3022.

[31] Lapini G, Zippo M, Tirone G. The Use of Electrostatic Charge Measurements as an Early Warning of Distress in Heavy-duty Gas Turbines. Proceedings of ASME Turbo Expo2001.

[32] Powrie H. Use of electrostatic technology for aero engine oil system monitoring. Aerospace Conference Proceedings: IEEE; 2000.p.57-71.

[33] Harvey T, Wood R, Denuault G, Powrie H. Effect of oil quality on electrostatic charge generation and transport. Journal of electrostatics 2002;55(1):1-23.

[34] Harvey T, Wood R, Denuault G, Powrie H. Investigation of electrostatic charging mechanisms in oil lubricated tribo-contacts. Tribology International 2002;35(9):605-614.

[35] Harvey T, Morris S, Wang L, Wood R, Powrie H. Real-time monitoring of wear debris using electrostatic sensing techniques. Proceedings of the Institution of Mechanical Engineers, Part J: Journal of Engineering Tribology 2007;221(1):27-40.

[36] Morris S, Wood R, Harvey T, Powrie H. Electrostatic charge monitoring of unlubricated sliding wear of a bearing steel. Wear 2003;255(1):430-443.

[37] Sun J, Wood R, Wang L, Care I, Powrie H. Wear monitoring of bearing steel using electrostatic and acoustic emission techniques. Wear 2005;259(7):1482-1489.

[38] Harvey TJ, Wood RJK, Powrie HEG. Electrostatic wear monitoring of rolling element bearings. Wear 2007;263(7-12):1492-1501.

[39] Craig M, Harvey T, Wood R, Masuda K,
Kawabata M, Powrie H. Advanced condition monitoring of tapered roller bearings, Part 1. Tribology International 2009;42(11):1846-1856.

[40] Yan Y. Mass flow measurement of bulk solids in pneumatic pipelines. Measurement Science and Technology 1996;7:1687-1706.

[41] Wang L, Yan Y, Hu Y, Qian X. Rotational speed measurement using single and dual electrostatic sensors. IEEE Sensors Journal 2015;15(3):1784-1793.

[42] Tajdari T, Rahmat MFa, Wahab NA. New technique to measure particle size using electrostatic sensor. Journal of electrostatics 2014;72(2):120-128.

[43] Powrie HEG. Electrostatic Charge Generation Associated with Machinery Component Generation. Proceedings of IEEE Aerospace Conference. Montana, USA: IEEE; 2002.p.2927-2934.

[44] Wen Z, Zuo H, Li Y. Gas path debris electrostatic monitoring technology and experiment. Journal of Aerospace Power 2008;23(12):2321-2326.(In Chinese).

[45] Wen Z, Zuo H, Li Y. New Method for Aero Engine Gas Path Monitoring. Journal of Nanjing University of Aeronautics \& Astronautics 2009;41(2):248-252.(In Chinese).

[46] Lawton J, Weinberg FJ. Electrical aspects of combustion. London: Clarendon Press; 1969.

[47] Ulybyshev K. Electric structure of the combustion front in a homogeneous methane-air mixture. Fluid Dynamics 1998;33(1):1-4.

[48] Kidin N, Makhviladze G. Electric field of laminar flame with high degree of ionization. Combustion, Explosion, and Shock Waves 1976;12(6):763-767.

[49] Wen Z, Hou J, Jiang Z. Formation Mechanism Analysis and Detection of Charged Particles in an Aero-engine Gas Path. International Journal of Aeronautical and Space Sciences 2015;16(2):247-253.

[50] Omidvarborna H, Kumar A, Kim D-S. Recent studies on soot modeling for diesel combustion. Renewable and Sustainable Energy Reviews 
2015;48:635-647.

[51] Vatazhin A, Golentsov D, Likhter V, Shul'gin V. Electrical aspects of body disintegration in a gas dynamic flow. Fluid Dynamics 1999;34(4):516-520.

[52] Flagan RC, Seinfeld JH. Fundamentals of air pollution engineering. North Chelmsford: Courier Corporation; 2012.

[53] Vatazhin A, Ulybyshev K. Model of Formation of the Electric Current in Aircraft Jet Engine Ducts. Fluid Dynamics 2000;35(5):748-755.

[54] Sorokin A, Arnold F. Electrically charged small soot particles in the exhaust of an aircraft gas-turbine engine combustor: comparison of model and experiment. Atmospheric Environment 2004;38(17):2611-2618.

[55] Sorokin A, Arnold F. Organic positive ions in aircraft gas-turbine engine exhaust. Atmospheric Environment 2006;40(32):6077-6087.

[56] Sorokin A, Vancassel X, Mirabel P. Emission of ions and charged soot particles by aircraft engines. Atmospheric Chemistry and Physics 2003;3(2):325-334.

[57] Ren J, Shen J, Lu S. Science and technology of particle dispersion. Beijing: Chemical Industry Press; 2005.

[58] Wen Z, Ma X, Zuo H. Characteristics analysis and experiment verification of electrostatic sensor for aero-engine exhaust gas monitoring. Measurement 2014;47:633-644.

[59] Zhang Z. The time to reach electrostatic equilibrium of a conductor. Physics and Engineering 2003;12(2):20-21.

[60] Chen Z, Tang X, Hu Z, Yang Y. Investigations into sensing characteristics of circular thin-plate electrostatic sensors for gas path monitoring. Chinese Journal of Aeronautics 2014;27(4):812-820.

[61] Forfitt R. Gas path electrostatic sensor. United States Patents; 1998.

[62] Lin J, Chen Z-S, Hu Z, Yang Y-M, Tang X. Analytical and Numerical Investigations into Hemisphere-Shaped Electrostatic Sensors. Sensors 2014;14(8):14021-14037.
[63] Tang X, Chen Z, Li Y, Hu Z, Yang Y. Theoretical analysis and finite element method simulations on dynamic sensitivity of hemisphere-shaped electrostatic sensors. Advances in Mechanical Engineering 2016;8(8).

[64] Tang X, Hu Z, Chen ZS, Yang YM. Investigation into Spatial Sensitivity of Probe-Type Electrostatic Sensors for On-Line Condition Monitoring of Heat Engines. Applied Mechanics and Materials: Trans Tech Publ; 2013.p.817-822.

[65] Xu C, Wang S, Yan Y. Spatial selectivity of linear electrostatic sensor arrays for particle velocity measurement. IEEE Transactions on Instrumentation and Measurement 2013;62(1):167-176.

[66] Xu C, Li J, Gao H, Wang S. Investigations into sensing characteristics of electrostatic sensor arrays through computational modelling and practical experimentation. Journal of electrostatics 2012;70(1):60-71.

[67] Powrie H, Wood R, Harvey T, Wang L, Morris S. Electrostatic charge generation associated with machinery component deterioration. Aerospace Conference Proceedings: IEEE; 2002.p.2927-2934.

[68] Liu R, Zuo H, Sun J, Wang L. Simulation of Electrostatic Oil Line Sensing and validation using experimental results. Tribology International 2017;105:15-26.

[69] Xu C, Wang S, Tang G, Yang D, Zhou B. Sensing characteristics of electrostatic inductive sensor for flow parameters measurement of pneumatically conveyed particles. Journal of electrostatics 2007;65(9):582-592.

[70] Smythe WR. Static and dynamic electricity: McGraw-Hill New York; 1950.

[71] Wen Z, Yin X, Zuo H. Optimization Design and Placement of the Electrostatic Sensor in Aero-engines Gas Path. Mechanical Science and Technology for Aerospace Engineering 2013;32(1):140-145.(In Chinese).

[72] Wen Z, Hou J, Zuo H. Characteristics analysis and extraction method for electrostatic 
monitoring signal in aero-engines gas path. Journal of Vibration, Measurement and Diagnosis 2015; 35(3):453-458. (In Chinese).

[73] Addabbo T, Fort A, Garbin R, Mugnaini M, Rocchi S, Vignoli V. Theoretical characterization of a gas path debris detection monitoring system based on electrostatic sensors and charge amplifiers. Measurement 2015; 64:138-146.

[74] Addabbo T, Fort A, Mugnaini M, Rocchi S, Vignoli V, Garbin R. Theoretical modeling of an electrostatic Gas-Path debris detection system with experimental validation. Sensors Applications Symposium (SAS), 2015 IEEE: IEEE; 2015.p.1-5.

[75] Chang CS, Jin J, Kumar S, Su Q, Hoshino T, Hanai M, et al. Denoising of partial discharge signals in wavelet packets domain. IEE Proceedings-Science, Measurement and Technology 2005; 152(3):129-140.

[76] Donoho DL. De-noise via soft threshold. IEEE Transform, Information Theory 1995;41(3): 613-627.

[77] Mallat SG. A theory for multiresolution signal decomposition: the wavelet representation. IEEE transactions on pattern analysis and machine intelligence 1989;11(7):674-693.

[78] Hoseini M, Zuo M. Gearbox Fault Diagnosis Using Two-Dimensional Wavelet Transform. Engineering Asset Management 2011: Springer; 2011. 643-653.

[79] Meng L, Xiang J, Zhong Y, Song W. Fault diagnosis of rolling bearing based on second generation wavelet denoising and morphological filter. Journal of Mechanical Science and Technology 2015;29(8):3121-3129.

[80] He W, Zi Y, Chen B, Wang S, He Z. Tunable Q-factor wavelet transform denoising with neighboring coefficients and its application to rotating machinery fault diagnosis. Science China Technological Sciences 2013;56(8):1956-1965.

[81] Lahmiri S. Comparative study of ECG signal denoising by wavelet thresholding in empirical and variational mode decomposition domains. Healthcare technology letters; 1(3):104.

[82] Wen Z, Zhao X. A hybrid de-noising method based on wavelet and median filter for aero-engines gas path electrostatic monitoring. 2011 International Conference on Graphic and Image Processing: International Society for Optics and Photonics; 2011.p. 82852G-82852G-82857.

[83] Wen Z, Zuo H. Electrostatic Monitoring Signal Processing Method Based on ICA for Aero-engines Gas Path. Mechanical Science and Technology for Aerospace Engineering 2011;30(11):1872-1876.(In Chinese).

[84] Wen Z, Hou J, Zuo H. Performance monitoring technology based on electrostatic for Aero-Engines. Aviation Maintenance \& Engineering. 2014;3:79-82.(in Chinese).

[85] Vatazhin AB, Golentsov DA, Likhter VA, Shulgin VI. Aircraft engine state nonobstructive electrostatic monitoring theoretical and laboratory modelling. Journal of Electrostiacs 1997;40(41):711-716.

[86] Couch R, Fowler R, Rossbach D, Vopalensky R. Apparatus for indicating the impending failure of a jet engine. U.S. Patents; 1973.

[87] Couch RP. Detecting abnormal turbine engine deterioration using electrostatic methods. Journal of Aircraft 1978;15(10):692-695.

[88] Sun J, Zuo H, Liu P, Wen Z. Experimental study on engine gas-path component fault monitoring using exhaust gas electrostatic signal. Measurement Science and Technology 2013;24(12):125107.

[89] Muir D, Howe B. In-line oil debris monitor (ODM) for the advanced tactical fighter engine. DTIC Document; 1996.

[90] Howe B, Muir D. In-line oil debris monitor (ODM) for helicopter gearbox condition assessment. DTIC Document; 1998.

[91] Miller JL, Kitaljevich D. In-line oil debris monitor for aircraft engine condition assessment. 2000 IEEE Aerospace Conference Proceedings: IEEE; 2000.p.49-56. 
[92] Masom R. The development, proving and application of an in-line metal particle detector (MPD). British Journal of Non-Destructive Testing 1985;27(3):159-166.

[93] Tasbaz O, Wood R, Browne M, Powrie H, Denuault G. Electrostatic monitoring of oil lubricated sliding point contacts for early detection of scuffing. Wear 1999;230(1):86-97.

[94] Morris S, Wood RJK, Harvey TJ. Use of Electrostatic Charge Monitoring for Early Detection of Adhesive Wear in Oil Lubricated Contacts. Transactions of the ASME 2002.p.288-296.

[95] Wen Z, Yin X, Jiang Z. Applications of electrostatic sensor for wear debris detecting in the lubricating oil. Journal of the Institution of Engineers (India): Series C 2013; 94(3): 281-286.

[96] Powrie H, Fisher C, Tasbaz O, Wood R. Performance of an electrostatic oil monitoring system during an FZG gear scuffing test. International Conference on Condition Monitoring1999.p.145-155.

[97] Gajewski J. Continuous non-contact measurement of electric charges of solid particles in pipes of pneumatic transport. I. Physical and mathematical models of a method. Industry Applications Society Annual Meeting: IEEE; 1989.p.1958-1963.

[98] Yan Y, Byrne B, Woodhead S, Coulthard J. Velocity measurement of pneumatically conveyed solids using electrodynamic sensors. Measurement Science and Technology 1995;6:515-537.

[99] Zhang P, Kong L, Huang XH. Mass flow measurement of pulverized material based on polarized charge. Proceedings of the
International Conference on Energy Conversity of Science and Technology Publishing Company2001.p.298-301.

[100] Woodhead SR, Denham JC, Armour-Chelu DI. Electrostatic sensors applied to the measurement of electric charge transfer in gas-solids pipelines. Journal of Physics: Conference Series, Institute of Physics Publishing; 2005.p.108-112.

[101] Gajewski JB. Non-contact electrostatic flow probes for measuring the flow rate and charge in the two-phase gas-solids flows. Chemical Engineering Science 2006;61(7):2262-2270.

[102] Matsusaka S, Masuda H. Simultaneous measurement of mass flow rate and charge-to-mass ratio of particles in gas-solids pipe flow. Chemical Engineering Science 2006;61(7):2254-2261.

[103] Zhang W, Yan Y, Yang Y, Wang J. Measurement of Flow Characteristics in a Bubbling Fluidized Bed Using Electrostatic Sensor Arrays. IEEE Transactions on Instrumentation and Measurement 2016;65(3):703-712.

[104] Coombes JR, Yan Y. Measurement of Velocity and Concentration Profiles of Pneumatically Conveyed Particles Using an Electrostatic Sensor Array. IEEE Transactions on Instrumentation and Measurement 2016;65(5): 1139-1148. 